\title{
The connection between shape and stellar population in early-type galaxies
}

\author{
Mauro D'Onofrio and the WINGS team \\ Department of Physics and Astronomy, University of Padova, \\ Vicolo Osservatorio 3, 35122 Padova, Italy \\ email: mauro.donofrio@unipd.it
}

\begin{abstract}
We comment on the observed connection between the shape of the photometric profiles and the stellar population content in early-type galaxies.
\end{abstract}

Keywords. galaxies: elliptical and lenticular, cD - galaxies: evolution - galaxies: formation

\section{Link with the Fundamental Plane}

Early-type galaxies (ETGs) are virialized stellar systems, so that a relation between their mass and structural properties (radius and velocity dispersion) is expected (the so called Virial Plane, VP). Observations show instead that ETGs share the Fundamental Plane (FP) which appears tilted with respect to the VP. By combining the VP and FP eqs. one can get (see, D'onofrio et al. (2013) for details):

$$
\log (M / L)-\log \left(K_{V}\right)=(2-a) \log (\sigma)+b\left(\langle\mu\rangle_{e}-M_{\odot}^{\lambda}-21.572\right)-\log (2 \pi G)-c
$$

where $K_{V}=1 / k_{v} k_{r}$ parametrizes our ignorance of the galaxy structure and dynamics $\left(\left\langle V^{2}\right\rangle=k_{v} \sigma^{2}\right.$ and $\left.\langle R\rangle=k_{r} R_{e}\right)$. Eq.1.1 already tell us that a connection between structural parameters and stellar population content must be in place.

The classical interpretation of the FP tilt is based on the hypothesis that $K_{V}=$ const and $(M / L) \propto M^{\alpha}$ (see e.g., Cappellari et al. 2013). However, D'onofrio et al. (2013) have demonstrated that $K_{V} \propto n$, where $n$ is the Sersic parameter determining the shape of the light profiles of ETGs.

The proof that behind the FP tilt there is the connection between the photometric shape of the light profiles and the stellar population comes from eq.1.1 that can be transformed into a relation between $\log (M / L)-\log (n)-\log (M)$ which has been observed to exist (see, D'onofrio et al. 2011).

In conclusion, we claim that the shape of the light profiles and the stellar population in ETGs are connected and this connection is at the origin of the FP tilt. This link between structure and stellar population in ETGS can be explained by the multiple dry merging capture of small galaxies. Evidence is growing in fact that dry merging events produce a change in the Sersic index of galaxies while little effects on total mass and stellar population $(M / L)$ (see e.g., Hiltz et al. 2013).

\section{References}

Cappellari, M., et al. 2013, MNRAS, 432, 1709

D'Onofrio, M., et al. 2011, ApJ, 727, L6

D'Onofrio, M., et al. 2013, MNRAS, 435, 45

Hiltz, M., et al. 2013, MNRAS, 429, 2924 